\title{
Resultados da implantação de um algoritmo para terapia nutricional enteral em crianças e adolescentes com câncer
}

\author{
Outcomes of the implementation of an enteral \\ nutrition algorithm in children and \\ adolescents with cancer
}

Adriana GARÓFOLO'

Priscila Santos MAIA ${ }^{1}$

Antonio Sérgio PETRILLI'

Fábio ANCONA-LOPEZ ${ }^{1}$

RE S U M O

\section{Objetivo}

Descrever o algoritmo e os resultados globais após sua implantação.

\section{Métodos}

Ensaio clínico randomizado realizado com pacientes com câncer e desnutrição. O acompanhamento foi guiado por algoritmo, e desnutridos leves eram randomizados para suplementação oral industrializada ou artesanal, reavaliados após três, oito e doze semanas. Dependendo da resposta no grupo suplementação oral industrializada, indicava-se suplemento industrializado por sonda, mantinha-se suplementação oral industrializada ou ela era suspensa. O grupo suplementação oral artesanal com resposta positiva permaneceu com a mesma orientação; se negativa iniciou com suplementação oral industrializada. Nos desnutridos graves, resposta negativa indicou suplemento industrializado por sonda, positiva manteve suplementação oral industrializada ou ela era suspensa no caso de eutrofia. A avaliação das respostas foi realizada por meio do escore-Z de peso para estatura, índice de massa corpórea, prega cutânea triciptal e circunferências.

\section{Resultados}

Cento e dezessete de 141 pacientes completaram três semanas iniciais; 58 desnutridos grave e 59 leve. Evolução nutricional positiva ocorreu em $41 \%$ dos graves, em $97 \%$ dos leves com suplementação oral industrializada e em 77\% dos desnutridos leves com suplementação oral artesanal. Dos 117, 42 tiveram indicação de suplemento industrializado por sonda; 23 usaram e 19 recusaram, mantendo suplementação oral industrializada. Pacientes

\footnotetext{
1 Universidade Federal de São Paulo, Escola Paulista de Medicina, Instituto de Oncologia Pediátrica, Departamento de Pediatria, Setor de Nutrição Clinica. R. Botucatu, 743, 04023-062, São Paulo, SP, Brasil. Correspondência para/Correspondence to: A. GARÓFOLO. E-mail: <adrigarofolo@hotmail.com>.
} 
716 | A. GARÓFOLO et al.

que usaram suplemento industrializado por sonda apresentaram consumo de suplemento superior aos demais; o consumo de suplemento industrializado por sonda também foi superior quando comparado ao período anterior com suplementação oral industrializada nos mesmos pacientes. O uso de suplemento industrializado por sonda demonstrou melhorar o estado nutricional, com associação positiva entre sua duração e evolução nutricional.

\section{Conclusão}

Suplementação oral industrializada reduziu o deficit nutricional, principalmente em desnutridos leves. Os resultados sugerem que a suplemento industrializado por sonda favoreceu a recuperação nutricional, principalmente com o uso mais prolongado.

Termos de indexação: Algoritmo. Neoplasias. Nutrição enteral. Terapia nutricional.

\section{A B S T R A C T}

\section{Objective}

This study aimed to describe the algorithm and the global results after its implementation.

\section{Methods}

This was a randomized clinical trial done with malnourished cancer patients. Follow-up followed an algorithm and patients with mild malnutrition were randomized to receive store-bought or homemade oral supplementation. The patients were reassessed after three, eight and twelve weeks. Depending on how the group supplemented with store-bought supplements responded, the supplementation was either continued orally, by tube-feeding or discontinued. The group receiving homemade supplementation either continued on it if the response was positive or received store-bought oral supplementation if the response was negative. The severely malnourished patients either received store-bought supplementation by feeding tube or orally, or it was discontinued if an adequate nutritional status was reached. The patients' responses to supplementation were determined by weight-for-height Z-scores, body mass indices, triceps skinfold thicknesses and circumferences.

\section{Results}

One hundred and seventeen out of 141 patients completed the first three weeks; 58 were severely malnourished and 59 were mildly malnourished. The nutritional status of $41 \%$ of the severely malnourished patients and $97 \%$ of the mildly malnourished patients receiving store-bought supplement orally improved. The nutritional status of $77 \%$ of the mildly malnourished patients receiving homemade supplement orally also improved. Of the 117 patients, 42 had to be tube-fed; of these, 23 accepted and 19 refused tube feeding and continued taking store-bought supplement orally. Consumption of store-bought supplement was higher in tube-fed patients than in orally-fed patients. Consumption also increased as orally-fed patients became tube-fed. Store-bought supplements given by tube improved nutritional status, showing a positive association between duration of tube feeding and nutritional status.

\section{Conclusion}

Store-bought oral supplements improved the nutrient intake of malnourished patients, especially those who were mildly malnourished. The results also suggest that store-bought supplements given by tube improve nutritional status, especially as duration of tube feeding increases.

Indexing terms: Algorithm. Neoplasms. Enteral nutrition. Nutritional support.

\section{N T R O D U Ç Ã O}

O câncer infantil compreende uma pequena porcentagem de todas as neoplasias malignas humanas na maioria das populações, estimando-se uma incidência anual de cerca de duzentos mil casos em todo o mundo. Essa taxa de incidência tem crescido em torno de $1 \%$ ao ano, inversamente proporcional à taxa de mortali- dade, com expectativa de que, em 2010, um em cada 250 adultos seja sobrevivente de câncer pediátrico. Em ordem de incidência, as principais regiões do globo são Uganda, Croácia, Nova Zelândia, Dinamarca, EUA e Canadá, sendo, normalmente, superior no sexo masculino ${ }^{1,2}$.

Os principais diagnósticos do câncer na infância são as leucemias, seguidas pelos tumores 
cerebrais, linfomas, neuroblastomas, sarcomas de partes moles, tumor de Wilms, tumores ósseos e retinoblastoma'.

O câncer é uma doença catabólica, em que o tumor maligno atua de forma a consumir as reservas nutricionais do hospedeiro, levando ao prejuízo nutricional. Entretanto, alguns tumores estão mais associados com a desnutrição, principalmente os tumores sólidos, provavelmente pelo crescimento mais indolente ${ }^{3-5}$.

As alterações nutricionais também podem ocorrer em decorrência do tratamento. Entre elas, a desnutrição se destaca como o distúrbio mais importante, geralmente estando associada à intensidade da terapia antineoplásica, causando efeitos negativos sobre a função gastrintestinal, além de outros efeitos tóxicos. Os agentes quimioterápicos utilizados em altas doses, comumente, induzem à anorexia, náuseas e vômitos intensos, diarréia, constipação, má absorção de nutrientes intestinais e mucosites. Além disso, pode ocorrer dor no local do tumor que, indiretamente, pode interferir com a alimentação $0^{6,7}$. Apesar desses aspectos, estudos com o objetivo de avaliar resultados da terapia nutricional nesses pacientes são escassos no Brasil.

\section{Histórico da terapia nutricional no Instituto de Oncologia Pediátrica}

Durante os anos de 1998 e 2000, 145 crianças e adolescentes que iniciaram tratamento no Instituto de Oncologia Pediátrica (IOP) foram acompanhadas pela equipe de nutrição. A investigação de 79 crianças demonstrou $10 \%$ de Desnutrido (DEP) pelo escore-Z de peso para estatura, observando-se maior porcentagem entre as crianças com tumores cerebrais $(4 / 15 ; 27 \%)$ e neuroblastomas $(3 / 12 ; 25 \%)^{3}$. A mesma avaliação foi realizada com os adolescentes, segundo o Índice de Massa Corporal (IMC), registrando-se uma prevalência total de $32 \%$ de DEP, com maior porcentagem entre os osteossarcomas (13/28; 46\%) ${ }^{8}$.

As medidas de composição corporal por meio de Prega Cutânea Triciptal (PCT), Circunferência do Braço (CB) e Circunferência Muscular do Braço (CMB) demonstraram maior percentual de deficit nos portadores de tumores sólidos não hematológicos pela $\mathrm{CB}$ e CMB, quando comparados aos demais (leucemias e linfomas). A análise global também sugeriu maior percentual da PCT (40\%) e CB (35\%) quando comparada ao escore-Z de P/E ou IMC (19\%). O maior deficit nutricional em pacientes com tumores sólidos poderia estar associado ao catabolismo da doença, porém o uso de corticosteróides em pacientes com doenças hematológicas também aumenta esse risco. As diferenças observadas entre os métodos poderiam estar associadas com o peso do tumor e o uso de corticosteróides, bem como com a sensibilidade dos indicadores ${ }^{9}$.

De forma geral, o maior risco para desnutrição durante o tratamento está associado à terapia com múltiplas drogas quimioterápicas em altas doses e sua combinação com radioterapia, principalmente abdominal ou pélvica. Determinados protocolos de tratamento, principalmente para alguns tumores sólidos não hematológicos, fazem uso de terapia antineoplásica muito agressiva, aumentando o risco de DEP10.

Acompanhando 20 adolescentes portadores de osteossarcomas durante o mesmo período de estudo, observou-se aumento na prevalência de DEP de 40\% para 50\% durante a fase de indução do tratamento, após um programa de intervenção dietética. Um aumento semeIhante nas taxas de deficit ocorreu por meio da avaliação da PCT e da CMB. Provavelmente, esse efeito ocorreu como consequência do uso de drogas quimioterápicas que prejudicam a ingestão alimentar e promovem perdas nutricionais importantes por toxicidade dos medicamentos sobre o rim e o trato gastrintestinal, principalmente vômitos intensos ${ }^{11}$.

Diante desses dados, em 2000 propôs-se o primeiro protocolo de recuperação nutricional por meio de suplementação oral, utilizando-se suplemento industrializado. Esse estudo teve como objetivo avaliar a resposta nutricional por oito semanas de oferta do suplemento, concomitante à orientação dietética, durante um acom- 
panhamento semanal de pacientes com DEP grave ou leve. O estudo demonstrou, globalmente, que $18 \%$ dos pacientes corrigiram o peso e que as taxas de desnutrição caíram de 67\% para 33\% no final do programa. Entretanto, alguns grupos, como o de pacientes com tumores ósseos, demonstraram respostas menos animadoras, levando a crer na necessidade de novas propostas ${ }^{12}$.

Assim, após a análise desses resultados, em 2002 iniciou-se uma nova proposta, baseada na experiência no IOP e de alguns grupos internacionais, que recomendam a utilização de algoritmos para terapia nutricional. Esses algoritmos são baseados, principalmente, no estado nutricional, ingestão de energia e integridade do trato gastrintestinal desses pacientes e têm como objetivo direcionar a terapia nutricional, padronizando as condições de risco que necessitam de intervenções específicas, como suplementos orais, sondas, gastrostomias e nutrição parenteral ${ }^{13-15}$.

Desse modo, desenvolveu-se um algoritmo adaptado à realidade da nossa população, considerando aspectos sociais, econômicos (uso de suplemento artesanal) e culturais (indicação da sonda ocorre mais tardiamente do que nos algoritmos citados) dos pacientes, familiares e equipe clínica. O objetivo deste estudo foi descrever o algoritmo e mostrar os resultados globais obtidos após sua implantação.

\section{M É T O D O S}

O presente estudo foi desenvolvido no Instituto de Oncologia Pediátrica do Departamento de Pediatria da Escola Paulista de Medicina (Unifesp).

Crianças e adolescentes portadores de neoplasias malignas em tratamento oncológico durante o período de janeiro de 2002 a janeiro de 2004, cujo critério de inclusão foi a presença de déficit nutricional caracterizada pelas seguintes situações:

- IMC menor que o percentil 15 e maior que 5 (adolescentes) ou escore- $Z$ de Peso para
Estatura (P/E) menor que -1,0 e maior que - 2,0 desvios-padrão (crianças), que foram classificados como pacientes com DEP leve (esses pacientes são considerados sem déficit ponderal de acordo com a World Health Organization $(\mathrm{WHO})^{16}$. A classificação da WHO foi aplicada para os adultos jovens (acima de 18 anos).

- IMC abaixo do $5^{\circ}$ percentil ou escore-Z de P/E menor que -2,0 desvios-padrão que foram classificados com desnutrição grave. Esses pacientes são considerados desnutridos (moderado e grave) pela WHO. Os pacientes acima de 18 anos classificados como DEP moderado ou grave pela WHO foram incluídos como DEP grave ${ }^{16}$.

Os critérios excluíram portadores de doenças não secundárias ao câncer ou ao seu tratamento como síndrome da imunodeficiência adquirida, diabetes mellitus, cardiopatias, doenças pulmonares, doenças gastrintestinais, insuficiência renal ou hepática, doenças endocrinológicas e síndromes genéticas; pacientes com idade inferior a um ano; distúrbio de mastigação e/ou deglutição; pacientes em uso de terapia nutricional por sonda ou nutrição parenteral.

\section{Delineamento do estudo}

O estudo faz parte de uma pesquisa mais ampla, baseada em um algoritmo desenvolvido para decisão da terapia nutricional por via enteral, que incluiu suplementação por via oral e por sonda enteral. Para o presente estudo, três braços do algoritmo foram analisados: o primeiro foi um ensaio clínico randomizado, que utilizou Suplementação Oral Industrializada (SOI) ou Suplementação Oral Artesanal (SOA) como medida de intervenção nutricional. O segundo foi um ensaio clínico não controlado que utilizou apenas SOI e o terceiro foi um ensaio clínico controlado não randomizado, que utilizou SOI e Suplemento Industrializado por Sonda Nasoenteral (SIS).

No ensaio clínico randomizado ou aleatório os pacientes classificados com DEP leve foram randomizados para receberem SOI ou SOA 
(receita padronizada de suplemento com ingredientes dietéticos naturais). No ensaio clínico não controlado todos os pacientes que foram classificados com DEP grave receberam SOI.

O ensaio clínico controlado não randomizado incluiu somente os pacientes que tiveram indicação de SIS em qualquer uma das semanas de reavaliação, que serão descritas nos parágrafos seguintes.

O protocolo garantia que todos os pacientes fossem acompanhados prospectivamente (semanalmente) para se estudar a resposta nutricional com a oferta dos suplementos orais durante três semanas. Após esse período, todos foram reavaliados de acordo com o estado nutricional para serem classificados quanto sua resposta e, portanto, direcionados para um dos braços do protocolo (algoritmo).

Os pacientes com DEP grave que não apresentaram resposta positiva, ou seja, sem ganho ponderal ou ganho insuficiente após três semanas (resposta positiva considerou um ganho $\geq 0,3$ desvios-padrão no P/E na criança ou $\geq 3 \%$ da adequação do IMC no adolescente) ou que continuaram com DEP grave, nas semanas oito ou doze tiveram indicação de SIS (nova abordagem nutricional). SOI foi mantida para os pacientes com DEP grave que apresentaram evolução nutricional adequada e suspensa para aqueles que se tornaram eutróficos, mantendo-se o acompanhamento semanal e a orientação dietética, realizando-se novas avaliações nas semanas oito e doze. Nessas semanas, SIS foi indicada (nova abordagem nutricional) para aqueles que se mantiveram DEP grave e SOI foi suspensa para os eutróficos, mantendo-se a orientação dietética e o acompanhamento até completarem doze semanas. Para aqueles que continuavam com DEP leve, a suplementação oral (SOI ou SOA) era mantida da semana oito até a doze, quando se finalizava o acompanhamento (Figura 1).

Para o grupo com DEP leve, a SIS foi indicada quando os pacientes com SOl evoluíram para DEP grave. No caso de evolução adequada, eram seguidos até completarem doze semanas. Entre- tanto, aqueles que iniciaram com SOA e na semana três evoluíram para DEP grave tiveram indicação de iniciar com a SOI (nova abordagem nutricional) e foram acompanhados por mais três semanas, avaliados, então, de acordo com os mesmos critérios do grupo que recebeu SOI. Para os pacientes com SOI que apresentaram evolução adequada na semana três, novas reavaliações eram realizadas nas semanas oito e doze com o intuito de se avaliar a necessidade de SIS. Assim, nessas semanas, caso o paciente apresentasse DEP grave, a SIS era indicada (nova abordagem nutricional). Entretanto, se o paciente estivesse eutrófico, suspendia-se SOI e caso estivesse com DEP leve, mantinha-se o SOI na semana oito até a semana doze, quando se finalizava o acompanhamento (Figura 1).

Todos os pacientes com indicação de SIS foram submetidos a uma avaliação clínica por um oncologista pediátrico para sua colocação. A posição gástrica foi o primeiro local de escolha na indicação de sonda nesse protocolo, somente sendo alterada quando da indicação formal de sonda nasoduodenal ou nasojejunal. As situações que contraindicavam temporariamente a passagem da sonda eram: graus de toxicidade gastrintestinal 3 e 4; tumores com risco de obstrução durante o procedimento; tumores abdominais rechaçando estômago e/ou alças intestinais com risco de perfuração e sangramento; e baixa contagem de plaquetas (abaixo de 30.000 células $/ \mathrm{mm}^{3}$, que não resolvesse com infusão de plaquetas).

Pacientes que iniciaram o protocolo apresentando massa tumoral extensa, superestimando o peso e subestimando o déficit nutricional (avaliação clínica e por imagem e medição da circunferência abdominal), foram classificados e acompanhados pelos indicadores de PCT e CB, indicando-se a sonda quando abaixo do $5^{\circ}$ percentil.

\section{Padronização da terapia nutricional oral e por sonda}

O suplemento industrializado utilizado tanto por via oral como por sonda foi o Nutren Jr 
pó (<10 anos) e Nutren 1.0 pó ( $\geq 10$ anos) (Nestlé-Nutrição Clínica). A princípio, a diluição utilizada foi ajustada para obter-se uma quilocaloria por mililitro de dieta, podendo ser consumido pelo paciente puro ou misturado a outros alimentos, porém sem sofrer aquecimento. Entretanto, essa diluição poderia ser modificada em caso de necessidade de restrição de volume ou de concentração. O SOA foi composto por leite integral, sorvete, leite condensado, clara de ovo cozida e suplementação lipídica (óleo vegetal ou margarina); algumas vezes os pacientes misturavam frutas ou achocolatados em pó. O suplemento industrializado foi fornecido pela instituição ao paciente semanalmente, enquanto o suplemento artesanal, por conter ingredientes de uso domiciliar, não era fornecido. Entretanto, em algumas circunstâncias em que se detectavam maiores

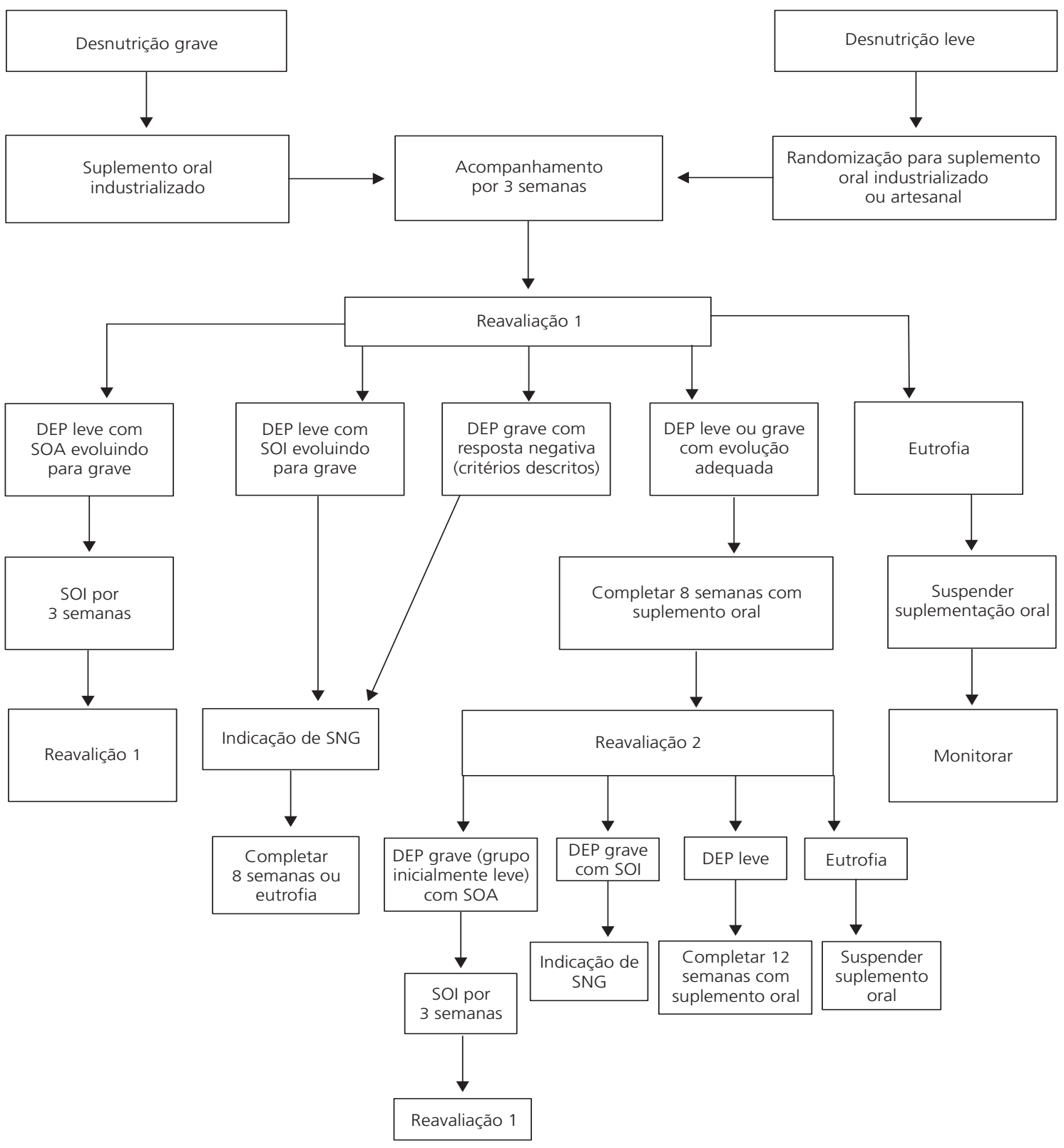

Figura 1. Algoritmo para decisão da terapia nutricional enteral.

Nota: SOI: suplemento oral industrializado; SOA: suplemento oral artesanal; DEP: desnutrição; SNG: sonda nasogástrica. 
dificuldades econômicas da família, alguns ingredientes poderiam ser doados por meio do serviço social ou então eram substituídos por outros, similares em energia e macronutrientes. Orientação nutricional dietética balanceada padrão foi realizada, estimulando-se o consumo de alimentos da cultura e do hábito familiar. A quantidade inicial de suplemento oferecida por via oral foi baseada em um teste prévio; porém, os pacientes foram motivados a consumirem acima dessa quantidade, quando aceitavam. Assim, o suplemento oral foi oferecido para cobrir $45 \%$ no mínimo, estimulando-se alcançar 100\%, dependendo da aceitação e tolerância do paciente, e o SIS para cobrir $100 \%$ da energia diária recomendada de acordo com as recomendações para pacientes oncológicos pediátricos ${ }^{13}$. O cálculo da necessidade total de energia diária recomendada (kcal/24 horas) foi ajustado semanalmente, considerando-se as mudanças no peso corporal.

A progressão do volume do SIS foi efetuada de acordo com o seguinte critério: iniciou-se com $50 \%$ da recomendação, aumentando-se para $75 \%$ em três dias consecutivos até alcançar 100\% em cinco dias, dependendo da tolerância gastrintestinal. Entretanto, essa quantidade poderia ser aumentada, caso o estado nutricional não demonstrasse evoluir na semana seguinte, sem que houvesse qualquer outro fator associado (infusão inadequada de dieta, perda da sonda, toxicidade gastrintestinal etc.).

Inicialmente, náuseas e vômitos foram minimizados com uma padronização de regime antiemético usada para os pacientes oncológicos da instituição durante o tratamento anticâncer, dependendo do protocolo de quimioterapia aplicado. Entretanto, quando a toxicidade limitava ou prejudicava a evolução do volume da dieta, alterações nas medicações ou na sua dose foram realizadas. Nos casos em que algum paciente apresentasse vômitos intratáveis acima de determinado volume, o volume da sonda foi reduzido para um nível previamente tolerado e aumentado quando a tolerância apresentasse melhora.

O SIS foi administrado no domicílio e durante as admissões hospitalares por meio de uma sonda do tipo duodenal de silicone e de pequeno calibre (6 a 12 F) (Fluocare; Support Nutrition). A sonda foi posicionada dentro do estômago e, após sua inserção, seu posicionamento era confirmado por Raios- $X$, inicialmente e semanalmente pela visualização da sonda marcada, junto à cavidade nasal. Quando havia suspeita de deslocamento da sonda, repetia-se a avaliação por Raios-X.

Durante as admissões no hospital, a dieta por sonda foi administrada por infusão contínua (24 horas) ou intermitente (oito vezes ao dia) em todos os pacientes, dependendo das condições clínicas. No domicílio, a rotina da sonda foi flexível e foi adaptada para as necessidades e possibilidades individuais dos pacientes. Alimentação noturna foi evitada devido à falta de disponibilidade de bombas de infusão no domicílio. Alimentação por via oral foi permitida ad libitum, mas não influenciou no volume da dieta orientado (SIS). Informações sobre o consumo da dieta oral bem como do suplemento por via oral ou sonda foram coletadas semanalmente durante as consultas com o nutricionista, por meio de um recordatório alimentar habitual. O consumo do suplemento foi investigado por meio da pesquisa do número de latas consumidas e do número de latas ainda em casa, abertas e fechadas.

Houve um grande empenho da equipe de nutrição com o intuito de alcançar a máxima adesão possível na administração do volume e horários prescritos. Os familiares foram orientados e encorajados semanalmente para oferecerem a suas crianças ou a seus adolescentes o volume e horários prescritos e thes foi permitido telefonar ou procurar o hospital todas as vezes que alguma dúvida ou problema ocorresse. Com a finalidade de compensar a perda de dieta por interrupções decorrentes dos procedimentos médicos, a taxa de infusão poderia ser temporariamente aumentada bem como alguns horários poderiam ser ajustados, quando tolerados. Os pacientes foram orientados a retornarem ao hospital caso a sonda fosse inadvertidamente ou acidentalmente sacada no domicílio, com o intuito de repassá-la. Esse 
procedimento foi realizado por enfermeiros treinados da equipe.

\section{Avaliação nutricional}

Foram realizadas medições de peso, estatura, PCT e CB por dois investigadores nutricionistas, treinados anteriormente para essa finalidade, seguindo os critérios de procedimentos descritos pela World Health Organization ${ }^{16}$ e Frisancho ${ }^{17}$. Dessas duas medições, calculou-se a circunferência muscular do braço, utilizando-se a seguinte equação: $C M B=C B-(P C T \times 0,314)$. Para a classificação do percentil, as variáveis foram interpretadas de acordo com as curvas de Frisancho ${ }^{17}$.

Para a interpretação dessas variáveis, utilizou-se o P/E e IMC como indicadores do estado nutricional em crianças e adolescentes respectivamente. Os padrões de referência para diagnóstico nutricional foram as curvas do NCHS e NHANES II, seguindo os critérios adotados pela WHO ${ }^{16}$.

A suplementação oral foi avaliada, levando-se em conta percentual mínimo da recomendação orientado (45\%) e recomendado (100\%). Para a avaliação do consumo de suplemento entre SOI e SIS, realizada no ensaio clínico não randomizado, foi efetuado o cálculo do Gasto Energético Total (GET), usando a seguinte equação: Gasto Energético Basal (GEB) × [fator recuperação, atividade $(1,2)$, doença $(1,3)$ e termogênese dos alimentos $(1,1)]$. O GEB (kcal/24h), de acordo com idade, sexo e peso, foi determinado pelas equações da Organização Mundial de Saúde (OMS), para pacientes menores de 15 anos e Harris Benedict para os maiores ${ }^{18,19}$. O fator recuperação usado para a correção da perda de peso foi determinado dividindo-se o peso ideal do paciente (P/E ou IMC) pelo atual ${ }^{20,21}$.

O teste do qui-quadrado foi utilizado para comparar as características categorizadas dos grupos: sexo, faixa etária (crianças e adolescentes), categoria de tumor (tumores sólidos e hemato- lógicos) e adesão dos pacientes às consultas nutricionais.

O teste T-pareado foi aplicado para comparar os resultados entre o período 1 (três semanas de SOI) e o período 2 (três semanas de SIS) no grupo sonda e comparar os indicadores nutricionais (mudanças percentuais na PCT, CB e CMB) da semana zero até a final de SIS. Quando a normalidade foi rejeitada, o teste de postos de Wilcoxon foi considerado.

O teste T para duas amostras foi aplicado com a finalidade de comparar os resultados entre os grupos SIS e SOI (controle que recusou a sonda e manteve SOI) no período 2. Quando a normalidade ou variâncias iguais foram rejeitadas, o teste de Mann' Whitney foi aplicado. Todos os testes estatísticos foram bicaudais.

Análise de regressão logística foi aplicada para determinar associação entre dados categóricos: uso de SOI e desfecho nutricional e aceitação do SOI maior ou menor que $80 \%$ e desfecho nutricional. Para essas análises, as variáveis de confundimento utilizadas foram idade, diagnóstico e escolaridade do cuidador principal. Outras variáveis de confundimento utilizadas foram grau de toxicidade gastrintestinal, episódio de hospitalização e tempo de hospitalização em dias.

Análise de regressão linear simples foi usada para estudar a associação das toxicidades gastrintestinais e desfechos nutricionais (mudanças no PCT, CB e CMB ideal) com o consumo do SIS da semana zero à última semana (final) de uso de sonda. A mesma análise foi realizada para avaliar a associação entre o uso de SIS (grupo sonda) ou por SOI (grupo-controle) e a intolerância gastrintestinal (vômitos e diarréia) no período 2 .

Análise de regressão múltipla foi aplicada para o estudo da associação entre a porcentagem de suplemento alcançado pela sonda (SIS) e os indicadores nutricionais da semana zero à semana final (mudanças nas diferenças percentuais da PCT, CB e CMB ideal) no grupo sonda e para avaliar a associação entre a duração da sonda com esses indicadores. Nível de escolaridade do cuida- 


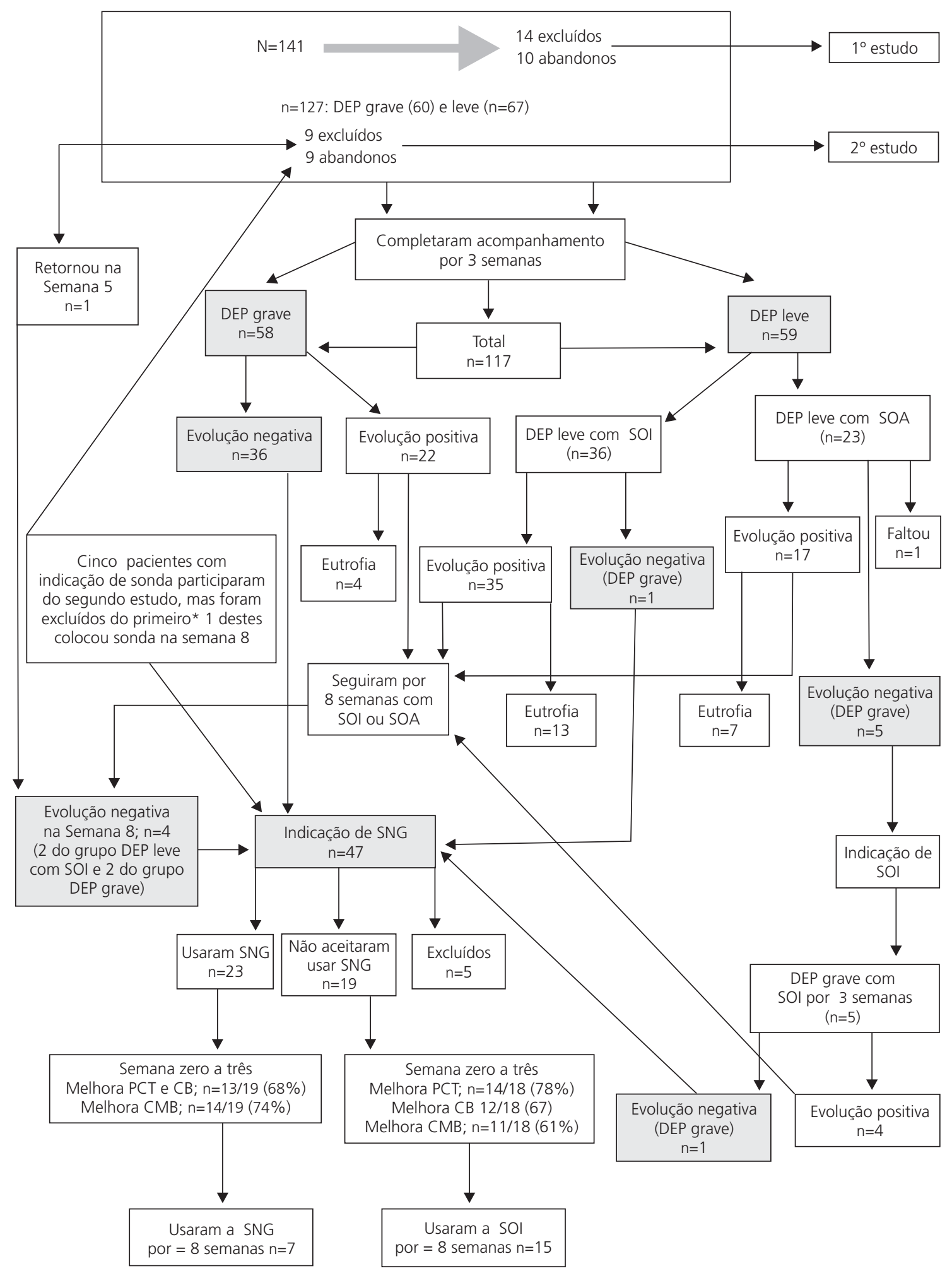

Figura 2. Resultados globais após a implantação do algoritmo.

Nota: *2 apresentaram peso superestimado pelo tumor, 2 colocaram sonda na semana zero e 1 necessitou de NPT exclusiva.

DEP: desnutrido; SOI: suplementação oral industrializada; SOA: suplementação oral artesanal; PCT: pregas cutâneas triciptal; CB: circunferência do braço; CMB: circunferência muscular do braço; SNG: sonda nasogástrica. 
dor principal, diagnósticos específicos e idade categorizada (criança ou adolescente) foram investigados como variáveis potenciais de confundimento. As análises foram realizadas pelo programa estatístico NCSS/PASS, versão $2000^{22}$.

O estudo recebeu aprovação do Comitê de Ética Médica da Escola Paulista de Medicina - UNIFESP - protocolo número 1097/02 (17/03/2003). Todos os pacientes foram esclarecidos sobre os riscos e benefícios das técnicas e intervenções e receberam uma carta de informação e consentimento para autorização do protocolo.

\section{RES U LTA D OS}

Cento e 17 pacientes de 141 completaram três semanas de acompanhamento. A Figura 2 mostra os resultados globais quanto à distribuição dos pacientes no estudo.

Os resultados globais demonstraram que 92\% dos pacientes aderiram ao protocolo, sem diferença estatisticamente significante quando se comparou SOI e SOA ( $90 \%$ vs $85 \%$ ), pacientes com DEP grave e leve ( $97 \%$ vs $86 \%$ ) e crianças e adolescentes (90\% vs $93 \%$ ).

\section{Ensaio clínico randomizado: SOI versus SOA}

A comparação entre os pacientes DEP leve com SOI e SOA, de acordo com sexo, idade e grupo de tumor, não demonstrou diferenças estatisticamente significantes. As características demográficas dos pacientes com SOI são $(n=36)$ : $42,5 \%$ do sexo feminino e $57,5 \%$ do masculino; $65 \%$ crianças e $35 \%$ adolescentes; $80 \%$ portadores de tumores sólidos (17,5\% tumores ósseos, $12,5 \%$ tumores de Wilms, $10 \%$ neuroblastomas, 7,5\% rabdomiossarcomas, $10 \%$ tumores do Sistema Nervoso Central (SNC), 7,5\% Tumores de Células Germinativas (TCG) e 15\% outros tumores menos frequentes) e 20\% tumores hematológicos (10\% leucemias e 10\% linfomas). Os pacientes do SOA apresentaram a seguinte ca- racterização ( $n=23): 48 \%$ dos pacientes do sexo feminino e $52 \%$ masculino; $74 \%$ eram crianças e 26\% adolescentes. Quanto aos diagnósticos, $67 \%$ eram portadores de tumores sólidos (22\% tumores ósseos, 7,4\% SNC, 7,4\% neuroblastomas, 3,7\% tumores de Wilms, 7,4\% rabdomiossarcomas, 7,4\% TCG e $11 \%$ outros tumores menos frequentes) e 33\% tumores hematológicos

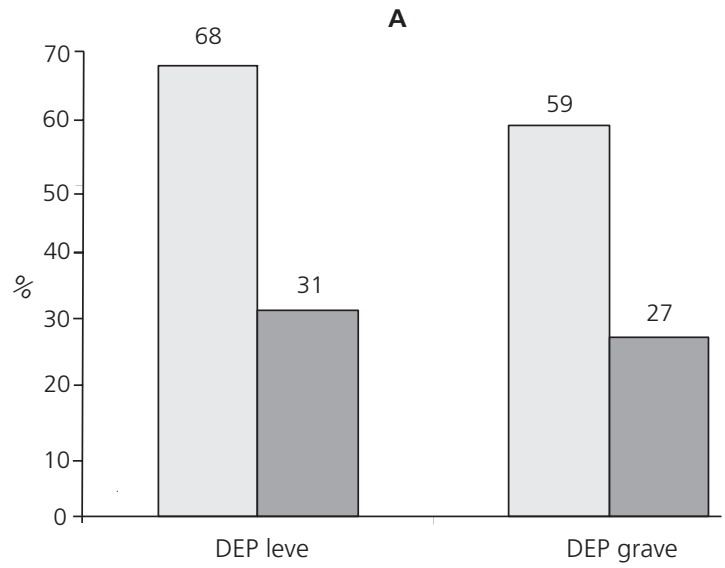

$45 \%$ recomendado $\square 100 \%$ recomendado

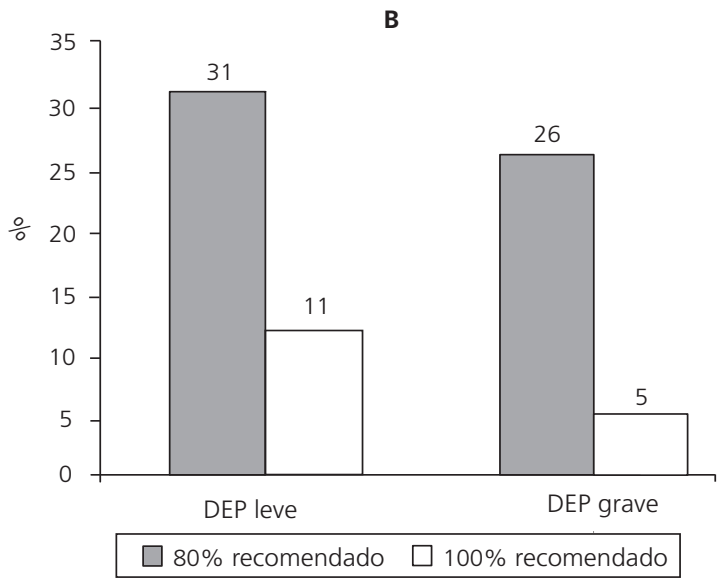

Figura 3. Resultados da aceitação do suplemento oral industrializado até a semana três. A) Porcentagem de aceitação sobre a orientação de $45 \%$ do recomendado e sobre $100 \%$ do recomendado (DEP leve; $n=36$ e DEP grave; $\mathrm{n}=57)$.B) Percentual de pacientes com aceitação igual ou superior a $80 \%$ e $100 \%$ do orientado até a semana três (DEP leve; $n=36$ e DEP grave; $n=57$. 
( $25,7 \%$ leucemias e $7,3 \%$ linfomas). As Figuras 3 e 4 mostram os resultados quanto à aceitação do SOI e a evolução do estado nutricional, respectivamente.

A análise de associação entre a resposta nutricional e a porcentagem de suplemento industrializado consumido não demonstrou associação entre os pacientes DEP leve com SOI.

\section{Ensaio clínico não controlado com SOI}

As características demográficas dos pacientes com DEP grave que receberam $\mathrm{SOI}$ foram ( $n=58$ ): $38 \%$ do sexo feminino e $62 \%$ masculino; $27 \%$ crianças e $73 \%$ adolescentes; $85 \%$ eram portadores de tumores sólidos (35\% tumores ósseos, $10 \%$ tumores do SNC, 10\% neuroblastomas, $7 \%$ rabdomiossarcomas, $7 \%$ tumores de Wilms, 3,3\% tumores de células germinativas (TCG) e $12 \%$ outros tumores menos frequentes) e $15 \%$ tumores hematológicos (5\% leucemias e $10 \%$ linfomas). As Figuras $3 \mathrm{~b}$ e $4 \mathrm{~b}$ mostram os resultados da aceitação do SOI e a evolução do estado nutricional.

A análise de associação entre a resposta nutricional e a porcentagem de suplemento industrializado consumido demonstrou correlação significante entre os pacientes DEP grave. A análise não ajustada demonstrou que os pacientes que consumiram SOI acima de $80 \%$ do prescrito tiveram menos necessidade de nova abordagem nutricional $(\mathrm{OR}=0,16 ; 95 \% \mathrm{Cl}=0,04-0.61$, $p=0,007$ ). No modelo ajustado, o consumo acima de $80 \%$ demonstrou uma tendência à significância na necessidade de nova abordagem nutricional ( $\mathrm{OR}=0,26 ; 95 \% \mathrm{Cl}=0,05-1.13$; $p=0,07$ ). Além disso, quanto maior o consumo de suplemento, menor a frequência de indicação de sonda (nova abordagem nutricional) $(p=0,005)$.

\section{Ensaio clínico controlado não randomizado: grupo SIS versus SOI}

Não houve diferenças estatisticamente significantes entre pacientes do SIS e pacientes do grupo SOI de acordo com sexo, idade, nível de escolaridade e grupo de tumor. As características Demográficas dos Pacientes com Sonda (SIS) são ( $n=23): 48,0 \%$ do sexo feminino e $52,0 \%$ masculino; $35,0 \%$ crianças e $65,0 \%$ adolescentes; $91,4 \%$ portadores de tumores sólidos (43,5\% tumores ósseos, 13,0\% neuroblastomas, $13,0 \%$ rabdomiossarcomas, $4,3 \%$ tumores do

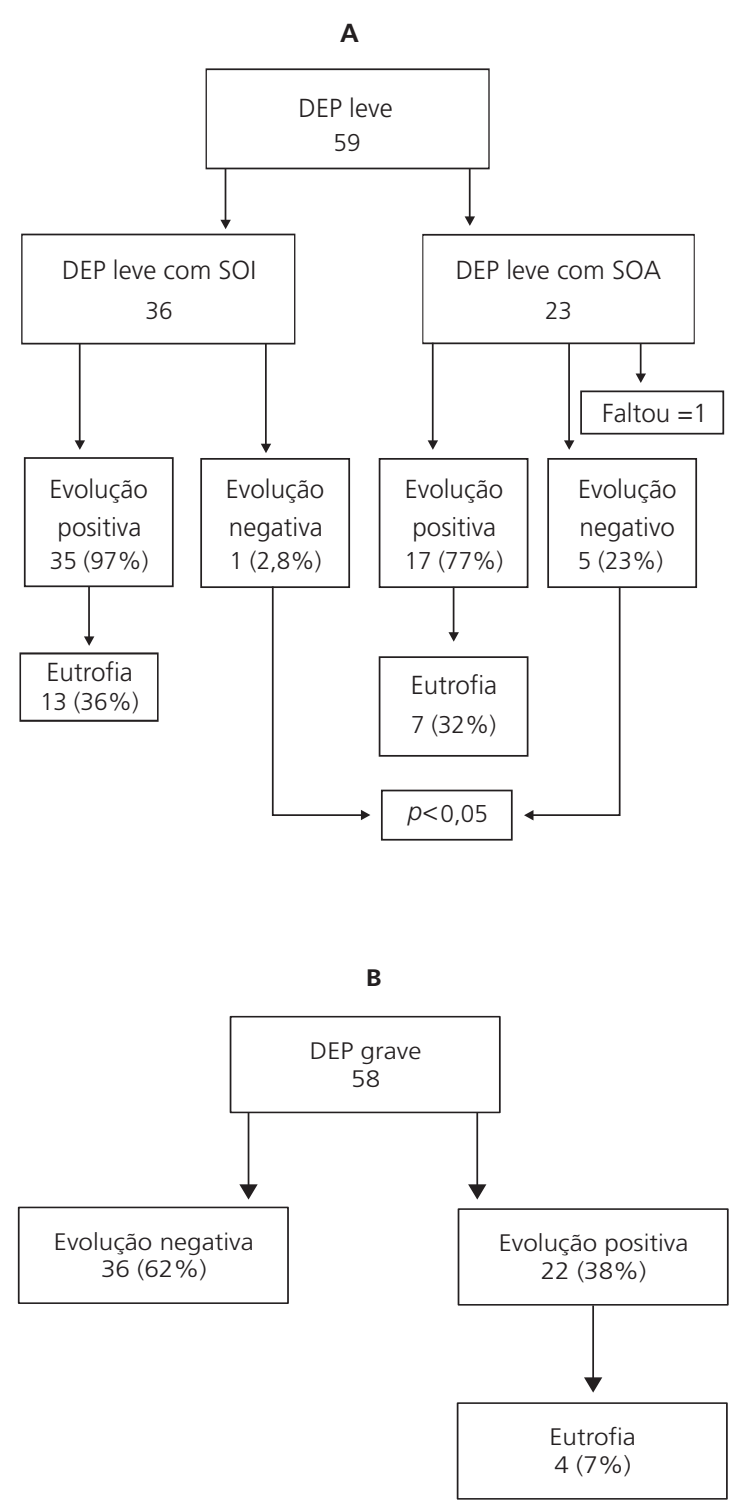

Figura 4. Porcentagem de pacientes de acordo com a evolução nutricional. A) DEP leve até a semana três.B) DEP grave até a semana três.

Nota: DEP: desnutrido; SOA: suplementação oral artesanal; SOI: suplementação oral industrializada. 
SNC, 4,3\% tumores de Wilms, 4,3\% Tumores de Células Germinativas (TCG) e 9,0\% outros tumores menos frequentes) e 8,6\% tumores hematológicos (4,3\% leucemias e 4,3\% linfomas). Os pacientes do grupo com SOI apresentaram a seguinte caracterização ( $n=19): 47,0 \%$ pacientes do sexo feminino e $47,0 \%$ masculino; $10,0 \%$ eram crianças e $90,0 \%$ adolescentes. Quanto aos diagnósticos, 84\% eram portadores de tumores sólidos (37,0\% tumores ósseos, 16,0\% SNC, 5,0\% tumores de Wilms, 5,0\% rabdomiossarcomas e $21,0 \%$ outros tumores menos frequentes) e $16,0 \%$ tumores hematológicos (10,0\% leucemias e $5,3 \%$ linfomas).

Alguns indicadores demonstraram diferenças de acordo com o estado nutricional na semana de indicação de sonda entre o grupo SIS e o grupo SOI para $C B(73,5 \%$ vs $76,9 \% ; p=0,06)$ e CMB $(77,9 \%$ vs $82,0 \%$; $p=0,08)$ e para IMC nos adolescentes $(71,9 \%$ vs $77,3 \% ; p=0,02)$.

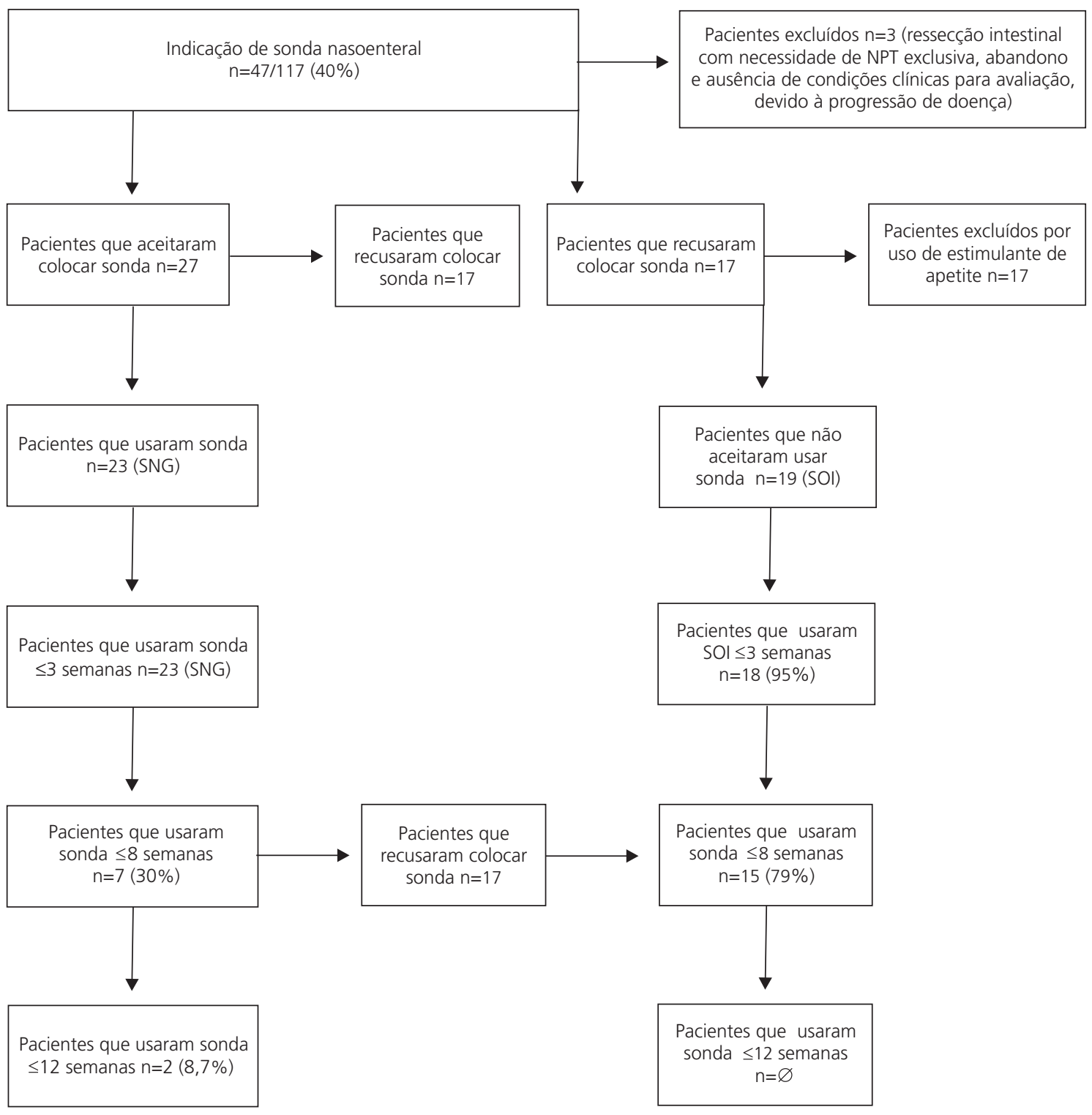

Figura 5. Resultados globais do uso de sonda.

Nota: SNG: sonda nasagástrica; DEP: desnutrido; SOI: suplementação oral industrializada; SOA: suplementação oral artesanal; NPT: nutrição parenteral total. 
Quarenta pacientes foram analisados quanto à porcentagem de $\mathrm{SOI}$ consumido antes da indicação de sonda (período 1); dois pacientes estavam em uso de sonda desde a semana zero. Essa análise demonstrou média e mediana de $51 \%$ Desvio-Padrão (DP) $=26$ e 56\% (0 - 100), respectivamente, da quantidade recomendada nesse período, sem diferença estatística entre os dois grupos: SIS vs SOI.

A média do consumo do suplemento durante o período 2 (após indicação da sonda) foi diferente entre o grupo SIS e o SOI $(p<0,00002$; teste de Mann' Whitney).

Comparando o grupo SIS no período 1 (quando recebendo suplemento por via oral) com o período 2 (recebendo suplemento pela sonda), observou-se diferença estatisticamente significante na quantidade consumida $(p=0,000004$; teste T paredo).

Não houve associação entre a porcentagem de suplemento consumido por sonda e os indicadores nutricionais.

O uso de SIS demonstrou evolução nutricional positiva entre as semanas zero até a semana final de uso: PCT zero $=50$ (31 - 100) e PCT final $=59(29-114), p=0,001$; CB: zero $=74(58-87)$ e final=79 (65 - 99), $p=0,0002 ; C M B$ zero $=77$ $(64-90)$ e final $=82(67-102), p=0,0004$ (Teste de Wilcoxon).

A Média (M) de uso de sonda no grupo SIS foi de 5,78 (DP=4,99) semanas (mediana $=5)$ e o uso de suplemento oral no grupo SOI foi de $8,16(\mathrm{DP}=2,03)$ semanas (mediana $=9$ ).

Foi possível observar uma associação positiva significante entre a duração do uso de sonda (SIS) e os indicadores do estado nutricional, demonstrando-se que quanto maior a duração do uso da sonda, maior aumento percentual dos indicadores nutricionais dos pacientes (semana zero e semana final): $P C T\left(R^{2}=0,55 ; r=2,52 ; p=0,0004\right)$, $C B\left(R^{2}=0,80 ; r=1,38 ; p=0,000001\right)$ e $C M B$ $\left(R^{2}=0,73 ; r=1,01 ; p=0,00003\right)$, ajustado para diagnóstico, idade e nível educacional.

Os resultados globais do uso de SIS vs SOI estão descritos na Figura 5.

\section{I S C U S S Ã O}

Os resultados deste estudo demonstraram 92\% de adesão global no grupo até a semana três de acompanhamento. Considerando que a falta de adesão ao tratamento oncológico é um problema apontado nos Estados Unidos em crianças e adolescentes ${ }^{23}$, essa porcentagem de adesão pode ser considerada como um resultado favorável. Quando os resultados de adesão às consultas foram comparados entre os grupos SOI e SOA, apesar de não haver diferença estatística entre eles, o grupo que recebeu o suplemento industrializado apresentou maior porcentagem na adesão. Isso poderia estar associado ao fato de a doação do SOI ter aumentado o interesse dos pacientes ao retorno às consultas. Os mesmos resultados ocorreram entre os pacientes com DEP grave e leve, podendo se supor que a gravidade da DEP poderia ter aumentado a preocupação em receber terapia nutricional.

Existe pouca informação a respeito da prática do uso de suplementos e sonda nasoenteral em crianças e adolescentes com câncer no Brasil. Em geral, as equipes médicas que tratam pacientes oncológicos pediátricos ainda são resistentes a procedimentos mais invasivos, como o uso de sondas enterais, que demonstrou ter uma baixa adesão, já que 45\% dos pacientes recusaram utilizá-la. É possível que a baixa conscientização da equipe clínica sobre a importância do estado nutricional no prognóstico tenha influenciado negativamente a decisão dos pacientes e seus cuidadores sobre a aceitação da terapia nutricional. Portanto, a conscientização geral da equipe com relação ao uso de sonda poderia ter facilitado a adesão dos pacientes. Provavelmente, essa dificuldade tenha ocorrido neste estudo por ser o primeiro protocolo com nutrição enteral por meio de sondas conduzido no IOP.

Um outro aspecto relacionado à adesão neste estudo foi o período de tempo que os pacientes permaneceram com sonda, que foi inferior ao período que o outro grupo se manteve com sol. A toxicidade por quimioterapia foi, prova- 
velmente, a principal causa de extubação neste estudo, devido aos vômitos intensos. Isso poderia estar relacionado aos protocolos antineoplásicos mais agressivos que incluem altas doses de drogas quimioterápicas como a cisplatina, um potente agente emetogênico.

Os resultados quanto à aceitação do suplemento em relação às três semanas iniciais demonstraram-se razoáveis, com médias de aproximadamente 60 a $70 \%$ do prescrito no protocolo (Figura 4a).

Neste estudo, a SOI nos indivíduos com DEP leve demonstrou uma resposta mais favorável do que a SOA, resgatando $97 \%$ do grupo, o que confirma sua importância na prevenção do agravo nutricional. Entretanto, a SOI em DEP grave não resgatou mais da metade do grupo, confirmando a necessidade de terapia nutricional mais especializada. Sabe-se que a DEP grave aumenta os riscos de complicações, o que leva ao maior risco de mortalidade para o paciente, bem como aumento dos custos hospitalares ${ }^{24}$.

Assim, este estudo mostra evidências da necessidade de se usar a sonda nasoenteral em grande porcentagem de pacientes com DEP grave.

Recentemente, o Comitê de Nutrição do Children's Oncology Group (COG) discutiu a necessidade de estudos para demonstrar a viabilidade, tolerância e aceitação do uso de sondas em crianças com câncer ${ }^{25}$. Bakish et al. ${ }^{26}$ observaram que os indivíduos que receberam alimentação por sonda apresentaram melhores respostas na recuperação nutricional do que aqueles com orientação de dieta por via oral ${ }^{26}$. Os resultados do presente estudo confirmam essa evidência, considerando os dados de evolução nutricional positiva, observados com o uso de sonda enteral. Tal melhoria foi maior do que aquela obtida nos períodos que os pacientes se mantiveram com suplementação oral.

Outros estudos também observaram resultados semelhantes, demonstrando adequação do estado nutricional atribuída ao uso de son$\mathrm{da}^{13,27-30 \text {. }}$
Vários estudos têm sugerido um período de uso de sonda de três a seis meses ${ }^{27-29,31}$. De fato, houve associação entre a duração da sonda e o estado nutricional neste estudo.

Em pacientes pediátricos com câncer, o uso de gastrostomia é preconizado quando há necessidade de terapia nutricional por sonda acima de três meses ${ }^{14,23}$. Por isso, neste estudo o período proposto com a sonda foi de oito semanas, apesar de alguns indivíduos requererem-na por tempo superior.

A nutrição enteral por sonda tem sido extensivamente preconizada para alimentação de crianças desnutridas com câncer ${ }^{27,32}$. Porém, interpretações dos estudos a esse respeito são difíceis, devido às limitações de delineamento: número de pacientes, ensaios não randomizados, diferentes diagnósticos, administração de nutrição parenteral concomitante e limitações dos métodos de avaliação nutricional. Além disso, tais estudos não apresentam informações a respeito da quantidade de suplemento consumido por sonda.

Considerações importantes para estudos futuros incluem aspectos como a necessidade de melhorar a oferta da energia total por meio do uso de bombas de infusão, principalmente durante o período noturno, administração protocolada de agentes anti-eméticos e pró-cinéticos e o uso de sondas nasoduodenais ou jejunais, bem como gastrostomias, quando necessário.

As principais limitações deste estudo foram: o número de pacientes, ausência de informação quanto à ingestão da dieta oral e a variabilidade da amostra quanto ao diagnóstico e idade. Outro aspecto foi a não randomização do grupo com indicação de sonda. Por outro lado, o presente estudo aplicou medidas nutricionais mais acuradas do que aquelas baseadas no peso.

$\mathrm{Na}$ literatura atual, poucos estudos com nutrição enteral realizaram um delineamento adequado. Vários são retrospectivos e todos apresentam grande variabilidade quanto ao diagnóstico e idade, e usaram o peso como único indicador nutricional. A maioria deles incluiu pequeno número de pacientes e não descreveu adequadamente aqueles que se recusaram a usar sonda e 
o critério para iniciá-la27-37. Considerando que a nutrição enteral tem menos risco do que a parenteral em crianças em tratamento anticâncer, mais estudos deste tema deveriam ser conduzidos a fim de elucidar os benefícios prognósticos desse método.

Assim, um estudo multicêntrico seria indicado com a finalidade de acompanhar um maior número de pacientes, o que permitiria uma análise e interpretação mais precisas dos resultados. Talvez, protocolos com nutrição enteral devessem ser incluídos como parte dos protocolos de tratamento anticâncer.

\section{O N CLUSÃ O}

Esses resultados sugerem que a adesão à nutrição enteral, quer por via oral, quer por sonda, deveria ser estimulada, principalmente por meio da equipe de oncologistas. Além disso, a nutrição enteral, principalmente por sonda, melhorou o estado nutricional sem demonstrar complicações gastrintestinais associadas a ela, enquanto a duração da terapia foi uma variável importante para o resultado nutricional.

\section{A GRADECIMENTO}

À Nestlé Nutrição Clínica pela parceria neste estudo.

\section{COLABORADORES}

A. GARÓFOLO escreveu o projeto, participou da coleta de dados, coordenou o desenvolvimento do estudo, discutiu e analisou os resultados e redigiu o texto do manuscrito. P.S. MAIA participou da coleta dos dados. A.S. PETRILLI e F.A. LOPES participaram da análise e discussão dos resultados

\section{REFERÊ N CIAS}

1. Rodrigues KM, Camargo. Diagnóstico precoce do câncer infantil: responsabilidade de todos. Rev Assoc Med Bras, 2003; 49(1):29-34.
2. Brasil. Ministério da Saúde. Câncer Pediátrico. Situação do Câncer no Brasil. Disponível em: $<$ www.inca.gov.br/sitacao>.

3. Garófolo A, Caran EM, Silva NS, Lopez FA. Prevalência de desnutrição em crianças com tumores sólidos. Braz J Nutr. 2005;18(2):193-200.

4. Ladas EJ, Sacks N, Meacham L, Henry D, Enriquez L, Lowry G, et al. A Multidisciplinary Review of Nutrition Considerations in the Pediatric Oncology Population: A Perspective From Children's Oncology Group. Nutr Clin Pract. 2005; 20(4):377-93.

5. Segura A, Pardo J, Jara C, Zugazabeitia L, Carulla J, Penas R, et al. An epidimiological evaluation of the prevalence of malnutrition in Spanish patients with locally advanced or metastatic cancer. Clin Nutr. 2005 24(5):801-14.

6. Rivadeneira DE, Evoy D, Fahey TJ, Lieberman MD, Daly JM. Nutritional support of the câncer patient. Cancer J Clin. 1998; 48(2):69-80.

7. Moynihan T, Kelly DG, Fich M J. To feed or not to feed: is that the right question? J Clin Oncol. 2005; 23(25):6256-9.

8. Garófolo A. Estado nutricional de crianças e adolescentes com câncer [tese]. São Paulo: Universidade Federal de São Paulo; 2000.

9. Garófolo A, Lopez FA, Petrilli AS. High prevalence of malnutrition in solid non-hematological cancer patients by using skinfold and circumference measures. São Paulo Med J. 2005b; 123(6):277-81.

10. Garófolo A, Lopez FA, Petrilli AS. Terapia nutricional em oncologia pediátrica. Pediatr Mod. 2001; 37(9): 413-27.

11. Garófolo A, Lopez FA, Petrilli AS. Acompanhamento do estado nutricional de pacientes com osteossarcoma. Acta Oncol Bras. 2002; 22(1): 233-7.

12. Garófolo A, Aragão KSM, Maia PS, Lopez FA, Petrilli AS. Suplementação oral e resposta do estado nutricional em crianças e adolescentes com câncer. Braz J Clin Nutr. 2002;17(1):1-8.

13. Bowman LC, Williams R, Sanders M, Smith K, Baker D, Gajjar A. Algorithm for nutritional support: experience of the metabolic and infusion support service of St. Jude Children's Research Hospital. Int J Cancer. 1998; 11:76-80S.

14. Children's Oncology Group Cancer Control - Nutrition Sub-Committee. Algorithm for nutrition intervention and categories of nutritional status in the pediatric oncology patient-references and resources. In: Children's Oncology Group. Symposium, 2004; Washington (DC).

15. Sala A, Antillon F, Pencharz P, Barr R. Nutritional status in children with cancer: a report from the AHOPCA workshop held in Guatemala city, August 
31-Septmber 5, 2004. Pediatric Blood Cancer. 2005; 45:230-36.

16. World Health Organization. Management of severe malnutrition: a manual for physicians and other senior health workers: malnutrition in adolescents and adults. Geneva: World Health Organization; 1999.

17. Frisancho AR. Antropometric standards for the assessment of growth and nutritional status. Michigan: The University of Michigan Press; 1993.

18. World Health Organization. Energy and protein requirements. Report of a joint FAO/WHO/UNU Expert Consultation. Geneva: World Health Organization; 1985. Technical Report Series 724.

19. Harris JA, Benedict F. A biometric study of basal metabolism in man. Washington (DC): Carnegie Institute of Washington; 1919.

20. St. Jude Children's Research Hospital. Clinical Nutrition Services. Clinical Practice Guidelines. Menphis (USA): St. Jude Children's Research Hospital; 2001. Section IV.

21. ASPEN Board of Directors and the Clinical Guidelines Task Force. Guidelines for the use of parenteral and enteral nutrition in adult and pediatric patients. JPEN J Parenter Enteral Nutr. 2002; 26(1 Suppl):1SA-138SA.

22. Dawson B, Trapp RG. Epi info [computer program] Version NCSS/PASS 2000: statistical software Dawson edition. Rio de Janeiro: McGraw-Hill; 2003.

23. Linnard-Palmer L, Kools S. Parents' refusal of medical treatment for cultural or religious beliefs: an ethnographic study of health care professionals' experiences. J Pediatr Oncol Nurs. 2005; 22(1):48-57.

24. Kyle UG, Pirlich M, Schuetz T, Lochs H, Pichard C. Is nutritional depletion by nutritional risk index (NRI) associated with increased length of stay (LOS): a population study. JPEN. 2004; 28 (2): 99-104.

25. Rogers PC, Melnick SJ, Ladas EJ, Halton J, Baillargeon J, Sacks N. Children's Oncology Group (COG) Nutrition Committee. Pediatr Blood Cancer. 2008; 50(Suppl 2):447-50.

26. Bakish J, Hargrave D, Tariq N, Laperriere N, Rutka JT, Bouffet E. Evaluation of dietetic intervention in children with medulloblastoma or supratentorial primitive neuroectodermal tumors. Cancer. 2003; 98(5):1014-20.

27. Papadopoulou A, Macdonald A, Williams MD, Darbyshike PJ, Booth IW. Enteral nutrition after bone marrow transplantation. Arch Dis Child. 1997; 77(2):131-6.

28. Hastings $Y$, White $M$, Young J. Enteral nutrition and bone marrow transplantation. J Pediatr Oncol Nurs. 2006; 23:103-10.

29. Langdana A, Tully N, Molloy E, Bourke B, O’Meia A. Intensive enteral nutrition support in pediatric bone marrow transplantation. Bone Marrow Transplantation. 2001; 27(7):741-6.

30. Barron MA, Ducan DS, Green GJ, Modrusan D, Connolly B, Chait P, et al. Efficacy and Safety of Radiologically Placed Gastrostomy Tubes in Paediatric Haematology/Oncology Patients. Med Pediatr Oncol. 2000; 34:177-82.

31. Aquino VM, Smyrl CB, Hagg R, McHard KM, Prestridge L, Sandler, ES. Enteral nutricional support by gastrostomy tube in children with cancer. J Pediatr. 1995; 127(1):58-62.

32. den Broeder E, Lippens RJ, van't Hof MA, Tolboom JJ, Sengers RCA, van den Berg AMJ, et al. Nasogastric tube feeding in children with cancer: the effect of two different formulas on weight, body composition, and serum protein concentrations. J Parent Ent Nutr. 2000; 24(6):351-60.

33. Pietsch JB, Ford C, Whitlock JA. Nasogastric tube feeding in children with high-risk cancer: a pilot study. J Pediatr Hematol Oncol. 1999; 21(2):111-4.

34. den Broeder E, Lippens RJ, van't Hof MA, Tolboom $J$, van Staveren WA, Hofman Z, et al. Effects of naso-gastric tube feeding on the nutritional status of children with cancer. Eur J Clin Nutr. 1998; 52(7): 494-500

35. Deswarte-Wallace J, Firouzbakhsh S, Finklestein JZ. Using research to change practice: enteral feedings for pediatric oncology patients. J Pediatr Oncol Nurs. 2001; 18(5):217-23.

36. Pedersen AM, Kok K, Petersen G, Nielsen $\mathrm{OH}$, Michaelsen KF, Schmiegelow K. Percutaneous endoscopic gastrostomy in children with cancer. Acta Paediatr. 1999; 88(8):849-52.

37. Sefcick A, Anderton D, Byrne JL, Teahon K, Russell $\mathrm{NH}$. Naso-jejunal feeding in allogeneic bone marrow transplant recipients: results of a pilot study. Bone Marrow Transplantat. 2001; 28(12): 1135-9.

Recebido em: 25/6/2007

Versão final reapresentada em: 4/5/2010 Aprovado em: 7/6/2010 